\title{
Una propuesta para trabajar la motricidad en educación infantil a través del aprendizaje inducido
}

\author{
A proposal for working motor function in child education through induced learning
}

\author{
Seminario de Educación Física en \\ Educación Infantil (E. U. Magisterio, de \\ Segovia, España) \\ Víctor M. López Pastor. (Coord.)* \\ María Ascensión Antón Miñambres \\ José Manuel García de la Puente \\ Roberto Hidalgo Sandín \\ Irene Llorente Lumbreras \\ Helena Pescador López \\ Laura Vázquez Santamaría*
}

En este artículo se presentan los planteamientos genéricos de un modelo didáctico para trabajar la motricidad en educación infantil en horario lectivo, que ha demostrado ser viable y adecuado, además de permitir una atención adecuada a la diversidad del alumnado en los treinta grupos con que hemos trabajado. La propuesta ha ido desarrollándose y perfeccionándose durante los últimos ocho años a través de un seminario permanente, que utiliza la investigación-acción como medio de perfeccionamiento profesional.

En el primer apartado se realiza la introducción a la temática, incluido un análisis breve del estado de la cuestión en España y explicando la fundamentación teórica y experimental de la propuesta. En el segundo se presentan las características principales del proyecto (las finalidades, los principios de procedimiento, la utilización de círculos de aprendizaje reflexivo, el modelo genérico de sesión, el sistema y los instrumentos de evaluación, los contenidos, y las instalaciones y materiales). En el tercer apartado se explica la organización y el funcionamiento del seminario permanente de Educación Física en Educación Infantil. El seminario permanente es la estructura básica en que se apoya el proceso de intervención e investigación en la práctica educativa. Por último, se presentan unas conclusiones sobre el desarrollo del proyecto.

\begin{abstract}
This article presents the generic approach of a didactic model for working motor function in child education during teaching hours. This model has proven its viability and suitability, aside from bringing about proper emphasis on the cultural diversity of the students from the thirty groups we have worked with. The proposal has been developed and improved over the last eight years, through an ongoing seminary based upon action-research as a means for professional training.

The first part, an introduction to the topic, includes a brief analysis of the state of the art in Spain, and explains the rationale and experimental grounds of the proposal. The second part presents the main features of the project (i. e., the goals, the acting principles, the use of reflective learning guilds, the generic session model, the evaluation system and tools, the contents, as well as the facilities and materials.) The third part explains the structure and dynamics of the ongoing seminary on Physical Education in Child Education, the basis of the intervention and research on educational practice process. Finally, some conclusions about the development of the project are withdrawn.
\end{abstract}

Key words: Physical Education in Child Education, induced learning, exploratory approach, action-research.

Fecha de recepción: 10 de julio de 2006

Fecha de aceptación: 12 de septiembre de 2006

\footnotetext{
* Doctor en Educación. Profesor de la E. U. Magisterio, de Segovia y coordinador del Seminario de Educación Física en Educación Infantil (E. U. Magisterio, de Segovia, España).

** Maestras y maestros especialistas en Educación Infantil y Educación Física. Actualmente trabajan en diferentes centros de Segovia, Madrid y Ávila y son miembros del Seminario de Educación Física en Educación Infantil (E. U. Magisterio, de Segovia, España). Direcciones de contacto: E. U. Magisterio, de Segovia.

P/ Colmenares, 1-40001-Segovia.Telf.: 921-46-31-95, vlopez@mpc.uva.es
} 


\section{Introducción}

Tradicionalmente la motricidad del alumnado de educación infantil era trabajada por las propias maestras tutoras, en mayor o menor grado, en función de los conocimientos que tuvieran, las instalaciones y los materiales de que dispusieran y la programación de cada semana. En la última década, cada vez es más habitual que se encargue de este cometido el profesorado de Educación Física (EF), siempre que tenga horario disponible después de haber cubierto las horas fijadas para el alumnado de primaria. En estas circunstancias, la motricidad infantil pasa a trabajarse en un horario fijo, que suele oscilar entre media hora y una hora a la semana. La mayoría del profesorado de educación infantil se refiere a este tipo de prácticas físicas con la expresión "psicomotricidad". También el profesorado de EF suele utilizar esta expresión, en parte para poder entenderse con las maestras-tutoras, en parte para diferenciarlas de las sesiones con el alumnado de primaria. Éstas últimas sí son "educación física", aunque a veces pueden ser también "gimnasia" o "deportes", según quién sea el interlocutor.

En España no existe una Referencias demasiado abundante ni actualizada que oriente de forma específica sobre el trabajo de la motricidad en estas etapas. Podemos diferenciar entre las obras propias de las corrientes psicomotricistas, publicadas en castellano en las décadas de 1960 a 1980 (Lapierre y Aucouturrier, 1974a, 1974b, 1977a, 1977b; Lapierre, 1984; Le Boulch, 1969, 1978; Vayer, 1983, 1985), las propuestas didácticas posteriores, pero considerablemente basadas en dichas corrientes (Blázquez y Ortega, 1985; Esparza y Petroli, 1984; Linares, 1989; Martínez, García y Montoro, 1988; Martínez y Núñez, 1978; Santiago, 1985; Stokoe y Harf, 1987), y las propuestas publicadas a partir de la implantación de la Logse, la mayor parte de ellas es más compleja y más elaborada (De Andrés, 1993; Aznar et al., 1998; Bueno y Manchón, 1990; Lleixa, 1991; Godall y Hospital, 2000a, 2000b, 2000c; Lázaro Lázaro, 2000; Lleixa, 2001; Mendiara, 1997). Tras unos años con pocas publicaciones especializadas, en los últimos años ha aparecido un grupo más numeroso de trabajos, la mayor parte de ellos con propuestas prácticas (Desrosiers y Tousignant, 2005; Escribá et al., 1999; Farreny y Román, 2005; Gil Madrona, 2003; González Rodríguez, 2001; López Pastor et al., 2004; Miraflores et al., 2006; Vila y Cardo, 2005), aunque no todos (Aucouturrier y Mende, 2004; Mendiara y Gil, 2003; Rigal, 2006; Pastor Pradillo, 2002).
En este artículo vamos a centrarnos en una propuesta concreta de trabajo para la etapa de educación infantil. Ha sido elaborada por un seminario permanente, a través de sucesivos ciclos de investigación-acción sobre su propia práctica educativa. La base de la propuesta es la utilización de una metodología de aprendizaje inducido, fundamentada principalmente en las propuestas y las experiencias desarrolladas por Blández (1995, 2000), sobre los ambientes de aprendizaje; por Mendiara (1997, 1999), sobre los espacios de acción-aventura; y por Vaca $(1995,2000)$, sobre el tratamiento pedagógico de lo corporal en educación infantil. También nos sentimos muy identificados con las propuestas de Lleixa (1991) y Godall y Hospital (2000a, 2000b, 2000c).

En forma resumida y genérica, podría decirse que cada una de las sesiones se basa en la disposición de un "entorno" de aprendizaje, que es un espacio concreto con los materiales puestos en una serie de "rincones", de modo que sugieran o permitan una serie de actividades y experiencias motrices a los niños. Intentamos ir ajustando dicho entorno con la actividad motriz que nosotros buscamos. En este espacio les dejamos que experimenten y jueguen libremente, intentando que se autorregulen con dos normas básicas (respetar a sus compañeros y el material). Tras esta primera fase solemos comenzar a intervenir. A veces porque entendemos que la actividad motriz del alumnado no se aproxima a las finalidades que habíamos previsto; otras, para hacer evolucionar su actividad, su control y su competencia motriz; otras veces, porque buscamos el juego colectivo, para evolucionar en su relación con los demás e ir aprendiendo a trabajar en grupo.

Como puede comprobarse, se parte de la motricidad espontánea del alumnado para derivarla posteriormente hacia un mejor desarrollo de las capacidades motrices y corporales, así como una toma de conciencia de su corporalidad y sus posibilidades de movimiento. En todo este artículo iremos profundizando en estos aspectos.

\section{Planteamientos básicos de la propuesta. Cómo trabajar la motricidad en educación infantil a través del aprendizaje inducido}

En este apartado vamos a presentar las características más importantes de la propuesta, que son cuatro:

1. Las finalidades y los objetivos educativos que nos planteamos. 
2. Los principios de procedimiento que emanan de dichos objetivos y que sirven para guiar la práctica cotidiana y para evaluarla.

3. El círculo de aprendizaje reflexivo que utilizamos, tanto con el alumnado infantil como en nuestra labor docente.

4. La estructura de sesión y el sistema de evaluación que utilizamos, los contenidos trabajados y las instalaciones y materiales con que solemos contar.

Hemos intentado que todos estos aspectos muestren la mayor coherencia interna posible.

\section{Objetivos educativos}

Las personas que formamos parte del seminario permanente nos hemos puesto una serie de objetivos que intentamos cumplir en nuestro trabajo cotidiano con el alumnado de educación infantil:

- Llevar a cabo un tratamiento educativo de los ámbitos corporal y motor del alumnado de educación infantil, dentro de una concepción educativa integral que considere todos los ámbitos del niño y la niña: afectivo, social, corporal y cognitivo. Aunque trabajamos con la motricidad del alumnado, no podemos perder de vista que los diferentes ámbitos, facetas e inteligencias están funcionando en forma integrada y que deben ser tenidas en consideración.

- Posibilitar la intervención del alumnado de los últimos cursos de Magisterio interesados y preocupados en esta temática, así como abrir un espacio para el trabajo colaborativo entre maestras y maestros de diferentes especialidades. Cuando varias personas con diferente formación colaboran para trabajar sobre una misma realidad educativa, la intervención en la misma suele verse enriquecida de modo considerable.

- Favorecer la colaboración entre el profesorado de educación infantil, el profesorado en formación inicial (alumnado E. U. Magisterio) y el profesorado encargado de esta formación inicial. Entendemos que es importante que se establezcan cauces de comunicación y relación entre la formación inicial del profesorado y la formación permanente, entre práctica y teoría, entre formación y perfeccionamiento profesional, entre la escuela y la universidad.

- Crear dinámicas de trabajo colaborativo en ẹl aula entre diferentes profesores, de modo que nos acostumbremos a trabajar junto a otros compañeros, debido a que esto permite ofrecer un mayor apoyo al alumna- do y un mejor análisis posterior de las prácticas que se llevan a cabo. Este tipo de procesos colaborativos y reflexivos pueden mejorar notablemente la calidad de la práctica educativa y el perfeccionamiento de todo el profesorado implicado.

\section{Principios de actuación e intervención.}

\section{Un paso más allá de la mera "metodología"}

A continuación exponemos una serie de "principios de actuación" que nos sirven de ayuda, orientación y apoyo a la hora de decidir cómo intervenir en el aula, así como a la hora de evaluar la adecuación de dichas intervenciones y tomar decisiones para la próxima sesión. La utilización de este tipo de principios de actuación es propia de los modelos de currículo entendidos como proyecto y proceso y de los enfoques de La enseñanza para la comprensión (López, Monjas y Pérez, 2003). Nos hemos basado en los siguientes:

\section{Intentamos que la intervención educativa esté} centrada en el cumplimiento de los siguientes "criterios de calidad educativa"

Búsqueda de equilibrio entre el ámbito afectivo y el resto de ámbitos. Entendemos que en esta etapa educativa el ámbito afectivo es uno de los ejes clave sobre los cuales debe girar la intervención docente, pero que debe ser equilibrado con el resto de ámbitos de desarrollo para alcanzar una verdadera educación integral.

Búsqueda de equilibrio entre la escuela y la vida cotidiana. Entre la cultura propia de su entorno y su grupo social y la cultura escolar; entre la cultura y las prácticas corporales y motrices que está construyendo durante el día, y la que se aporta y reelabora en la escuela; entre la experiencia previa y el enriquecimiento del bagaje personal. La escuela y la vida cotidiana del alumnado deben ser un solo universo.

Búsqueda de equilibrio entre los intereses y los objetivos de las maestras y los del alumnado. Queremos llegar a conseguir un cierto equilibrio entre las necesidades y los intereses propios del alumnado y los del profesorado. El aprendizaje inducido a través del espacio y el material es una metodología que responde muy bien a este principio, pero no es la única estrategia que hay que poner en práctica.

Búsqueda de equilibrio entre los intereses individuales y los grupales. En esta etapa los niños manifiestan conductas claramente egocéntricas, en las cuales reclaman una atención individual. Entendemos que en parte hay que satisfacer dicha necesidad, pero tiene que 
ir aprendiendo que no está solo en el mundo, que forma parte de un grupo y que debemos encontrar un equilibrio razonable entre la atención individual y la acomodación a las necesidades del grupo; entre mis derechos como individuo y los derechos de los demás individuos con quienes convivo; entre el tiempo de trabajo individual y el tiempo de trabajo compartido y de relación con el otro y los otros.

\section{Creamos entornos de aprendizaje con el espacio y los materiales disponibles en el centro}

Posteriormente invitamos a los niños y a las niñas a jugar en dichos entornos, con las orientaciones básicas, más las orientaciones específicas que requiera cada uno de los rincones creados. La forma en que se pone el espacio y el material, junto con las indicaciones y los desafíos que se les proponen, induce el contenido motor a trabajar con el alumnado. La puesta y la utilización del material estarán condicionadas por los objetivos educativos del profesorado. La intervención educativa parte del juego libre exploratorio del alumnado, para hacerla evolucionar después a través de las intervenciones y orientaciones de las maestras.

\section{La coordinación con las maestras-tutoras}

Nos preocupa mucho el establecer relaciones de colaboración y comunicación entre el profesorado que lleva a cabo la actividad motriz y la maestra tutora de cada grupo, así como el intercambio de información sobre las características del alumnado y el proceso de enseñanza-aprendizaje que se realiza, de modo que las sesiones estén conectadas y coordinadas con dicho proceso.

\section{La organización de la práctica docente}

En el caso del seminario permanente, y gracias a que se cuenta con la colaboración de maestras en formación inicial, la intervención docente se realiza, de manera preferente, en grupos de tres personas. Cada una de ellas tiene una función específica durante la sesión: una desempeña el rol de maestra principal y es la encargada de dirigir la sesión; la segunda desempeña el rol de maestra de apoyo; mientras que la tercera se centra en realizar una narración de lo que ocurre en la sesión y/o de llevar a cabo la evaluación del alumnado. Las narraciones y una observación más detallada de las acciones del alumnado nos permiten realizar un proceso más fundamentado de reflexión sobre la acción, que nos ayuda a tomar decisiones más ajustadas en la siguiente sesión. Lógicamente, en las situaciones cotidianas de los colegios es complejo conseguir que haya tres personas en el

aula, pero no es tan difícil que haya dos, bien porque la tutora y la especialista trabajan juntas, o bien porque se busca la colaboración de otras personas (profesorado de apoyo, profesorado en formación inicial).

\section{Los ciclos de investigación-acción}

\section{en el seminario permanente}

Cada una de las sesiones ha sido planificada la semana anterior a llevarla a cabo a partir de lo analizado en las sesiones del seminario permanente. Del mismo modo, en el seminario se analiza a posteriori lo que ha ocurrido en cada sesión, de modo que van encadenándose ciclos de planificación-acción-análisis-planificación, que generan una espiral de investigación-acción durante el curso escolar. Cuando se cuenta con los permisos oportunos, se realizan también grabaciones en video y/o fotografías, que permiten hacer un análisis colectivo más sistemático y documentado. Los principios de actuación son un buen referente a la hora de evaluar los procesos de aprendizaje que están desarrollándose en las aulas, $y$ las decisiones que hay que tomar para ir avanzando de un modo coherente.

\section{Los círculos de aprendizaje reflexivo}

Tanto en nuestro trabajo directo con el alumnado, como en nuestro trabajo docente individual y colaborativo a través del seminario, intentamos trabajar con círculos de aprendizaje reflexivo (Kolb, 1984), debido a que constituye la dinámica más coherente y más lógica cuando se quiere comprender lo que se está haciendo y aprender en una forma consciente, compleja y profunda. Pueden recibir también el nombre de procesos reflexivos, ciclos de acción-reflexión, reflexión sobre la acción, entre otros. Lógicamente, guardan una estrecha relación con las dinámicas propias de la investigaciónacción, a través de ciclos y espirales. La estructura es sencilla, aunque puede presentar ligeras variantes:

Planificación

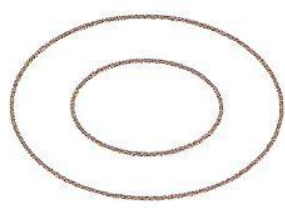

Acción

\section{Reflexión}

Gráfica 1. El círculo de aprendizaje reflexivo.

Como puede comprobarse en el siguiente punto, en que explicamos la estructura de sesión que utilizamos, desarrollamos estos círculos de aprendizaje reflexivo 
con el alumnado, en el trabajo de su motricidad. Entendemos que el trabajo de la motricidad infantil no puede estar basado en sesiones aisladas e inconexas entre sí, sino que debe existir una cierta continuación, una evolución, entre unas sesiones y otras.

Nuestro trabajo como docentes también está basado en este mismo tipo de procesos reflexivos, pero cambiando el foco de atención. En nuestro caso, los procesos reflexivos se centran en los procesos de aprendizaje de nuestro alumnado, en el proceso de enseñanza-aprendizaje que llevamos a cabo con cada grupo y en el proceso de aprendizaje y perfeccionamiento profesional que realizamos como docentes. Son, a su vez, los tres aspectos básicos de la evaluación formativa que llevamos a cabo.

\section{La estructura de sesión y sus implicaciones en la metodología de intervención}

Nuestra propuesta está basada de modo fundamental en los trabajos de Blández (1995, 2000), Mendiara (1997, 1999) y Vaca $(1995,2000)$ y, en una forma menos directa, por las propuestas de Lleixa (1991) y Godall y Hospital (2000a, 2000b, 2000c). Estas propuestas comparten muchas características; lógicamente, también tienen matices diferenciadores. En el caso de la estructura de sesión, las tres comparten una estructura muy similar en las tres grandes fases en que organizan la sesión, aunque presentan ciertas diferencias en el funcionamiento dentro de ellas. En el cuadro 1 se resume la estructura de sesión de las tres propuestas.

\begin{tabular}{llll}
\multicolumn{1}{c}{ Autor } & Parte inicial & \multicolumn{1}{c}{ Parte central } & \multicolumn{1}{c}{ Parte final } \\
$\begin{array}{l}\text { Mendiara } \\
(1997,1999)\end{array}$ & $\begin{array}{l}\text { Información } \\
\text { inicial }\end{array}$ & Juego activo & Verbalización final \\
\hline $\begin{array}{l}\text { Blández } \\
(1995,2000)\end{array}$ & Encuentro inicial & $\begin{array}{l}\text { Desarrollo de la } \\
\text { actividad }\end{array}$ & Puesta en común \\
\hline $\begin{array}{l}\text { Vaca } \\
(1995,2000)\end{array}$ & $\begin{array}{l}\text { Momento de } \\
\text { encuentro }\end{array}$ & $\begin{array}{l}\text { Momento de activi- } \\
\text { dad motriz }\end{array}$ & $\begin{array}{l}\text { Momento de } \\
\text { despedida }\end{array}$ \\
\hline
\end{tabular}

Cuadro 1. Comparación de las estructuras de sesión propuestas.

Del mismo modo, nuestra propuesta también divide la sesión en tres grandes partes: una asamblea inicial, una actividad motriz y una asamblea final. Cada una de estas partes se divide a su vez en una serie de fases, que pueden variar en función del contenido trabajado, el contexto, las reacciones de las niñas y los niños, los intereses que manifiestan, y/o las intenciones y los objetivos del profesorado. Habitualmente intentamos que una sesión evolucione como presentamos en el cuadro 2 , aunque no siempre es así. A veces se debe a que no logramos que evolucione como habíamos planificado, otras veces a que entendemos que es más convenien- te modificar la estructura para adecuarnos mejor a las características de cada contexto, grupo y sesión. La estructura de sesión habitual es la siguiente:

\footnotetext{
Asamblea inicial (de 5 a 10 minutos)

- Saludo

- Asamblea: recuerdo y conexión

- Recuerdo de la normativa básica y planteamiento de la actividad
}

Actividad motriz (de 30 a 45 minutos)

- Exploración libre

- Evolución de la actividad

- Paradas en corro durante la actividad motriz

- Trabajo en grupo

- Recogida de la sala

Asamblea final (de 5 a 10 minutos)

- Verbalización

- Despedida

- Representación gráfica

Cuadro 2. Esquema de la estructura de sesión.

Intentamos que la duración de la asamblea inicial esté entre los 5 y los 10 minutos, aunque puede haber alteraciones cuando las circunstancias lo requieren. $\mathrm{La}$ primera actividad que realizamos es un saludo. En la mayor parte de los casos, el saludo es individual, bien sea dando la mano, chocándola, dando un abrazo, etc., se trata de una actividad que puede presentar numerosas variantes y que suele evolucionar con el paso de las sesiones. Algunos profesores prefieren hacer un saludo colectivo, en algunas ocasiones, o todos los días. Es una opción bastante personal. En algunos casos, el grupo de maestras decide crear una rutina inicial a modo de saludo, con una breve canción motriz. Si hay vestuarios, previamente se han cambiado de calzado. Si no los hay, puede ser el momento de hacerlo, o bien de descalzarse. A continuación nos sentamos en corro y realizamos una asamblea. Sirve para repasar lo que han hecho en la sesión anterior, con dibujos o sin dibujos. También se recuerdan los nombres de los diferentes materiales que hay en el entorno y los juegos que pueden realizarse en cada uno de los rincones. Antes de salir a jugar, entre todos recordamos las normas que hay que respetar. Las básicas serán respetar a los compañeros y el material. A veces se introduce una para que cierto material pueda o no pueda utilizarse: por ejemplo, los aros.

A continuación llega la parte de la actividad motriz, que suele durar entre 30 y 45 minutos. La primera fase es la de juego y exploración libre. En esta fase es muy importante realizar una observación adecuada de los juegos del alumnado, debido a que nos muestran sus intereses, posibilidades, limitaciones y dificultades. Se trata de un aspecto clave para poder hacer evolucionar 
la sesión correctamente. La siguiente fase es muy dúctil, debido a que no siempre se da en forma clara ni en el mismo momento. A través de pequeñas intervenciones, preguntas y/o sugerencias, tratamos de hacer evolucionar la actividad motriz del alumnado. A partir de sus juegos exploratorios intentamos hacer progresar las habilidades y las competencias que muestran; o bien guiar la sesión hacia situaciones de interacción con los otros (trabajo por parejas o pequeños grupos). En cualquier momento de la actividad motriz puede ser necesario realizar una parada en corro. Se trata de momentos en que se detiene la actividad motriz para aclarar algunos aspectos de la misma, recordar alguna norma, plantear una nueva propuesta o una evolución de la anterior, cambiar de actividad o de agrupación, etc. Cerca de la mitad de la actividad motriz, o un poco más tarde, intentamos pasar a la fase de trabajo en grupo. A partir de las primeras intervenciones y paradas, se va intentando que la sesión avance hacia lo colectivo: primero a través del trabajo por parejas o grupos pequeños, para intentar avanzar en la segunda mitad de la sesión hacia el juego colectivo. Antes de pasar a la tercera y última parte de la sesión, solemos realizar la recogida de la sala. Si es con ayuda del alumnado, se hace antes de la verbalización o justo antes de la despedida. Si es sin las niñas y sin los niños, la hacemos mientras ellos están en el vestuario, o cuando se van al gimnasio.

A la tercera fase la denominamos asamblea final y suele durar entre 5 y 10 minutos. Primero nos sentamos en corro y realizamos una verbalización de las diferentes acciones y juegos que han llevado a cabo durante la sesión. Damos mucha importancia al hecho de que vayan aprendiendo a hablar por turnos y a pedir la palabra antes de hablar. Procuramos que todas las niñas y todos los niños hablen un poco cada día, o al menos que cada día sean unos diferentes, para que todos verbalicen sus acciones motrices. Tras la verbalización llega el momento de la despedida, que, al igual que en la primera parte, puede ser individual o colectiva. Si hay vestuarios, pasarán a cambiarse de zapatillas y/o ponerse el abrigo. Si no los hay, a calzarse. Estas fases pueden sufrir alguna variación dependiendo del funcionamiento de cada grupo de alumnos y de la instalación en que se realice la actividad. La última fase es la de representación gráfica. Al final de la verbalización solemos pedirles que hagan un dibujo de lo que más les haya gustado de la sesión y que nos lo traigan al día siguiente. Normalmente esta fase se hace ya en el aula, con la maestra tutora.

\section{Los contenidos que se van a trabajar}

Los contenidos que trabajamos durante la intervención educativa son los siguientes:

- Toma de conciencia de la propia corporalidad y las capacidades y las limitaciones motrices del alumnado.

- Los hábitos básicos de higiene, seguridad y vestimenta.

- Las implicaciones y las aportaciones de los aspectos corporal y motor en cada una de las áreas de conocimiento y experimentación del currículo de educación infantil.

- Los contenidos más propios de la intervención motriz en educación infantil: esquema corporal, estructuración y orientación espacio-temporal, expresión y dramatización, coordinación, dinámicas y habilidades físicas básicas, actividades grupales y cooperativas, ritmo y movimiento.

\section{Un sistema de evaluación orientado al mejoramiento y el aprendizaje}

En relación con la evaluación nos basamos en el sistema de evaluación formativa y compartida (López et al., 1999, 2006). Ello implica que nuestra evaluación va orientada al mejoramiento de las personas que estamos implicadas en el proceso. Evaluamos tres aspectos de nuestra intervención educativa: los procesos de aprendizaje del alumnado, el proceso de enseñanza-aprendizaje que se lleva a cabo y nuestra actuación docente. En cuanto a la metodología, nos basamos principalmente en dos dinámicas:

- En la propia realización de los ciclos de reflexión sobre la acción y la investigación-acción. Planificación previa de las sesiones, observación y realización de narraciones durante las mismas y análisis grupal tras su realización, con la consiguiente toma de decisiones sobre las siguientes intervenciones. Para llevar un registro más sistemático de este proceso, se hace la transcripción y la acumulación de las narraciones y los análisis llevados a cabo, de modo que puedan consultarse cuando sea necesario.

- La realización de fichas de seguimiento individual y grupal sobre los aspectos considerados más importantes en cada grupo de alumnos, a partir de las propuestas y experiencias de López et al. (1999, 2004). A continuación se presenta un ejemplo de ficha de seguimiento individual (cuadro 3). Está diseñada para poder utilizarse en forma genérica, durante todo el curso escolar, con un grupo de tres años. Como pue- 
de comprobarse, tiene 14 aspectos por evaluar, organizados en tres ámbitos (sociabilidad, motricidad y expresión). Pueden hacerse dos tipos de valoraciones: la primera, con una escala numérica o verbal (las siete columnas delgadas, tras los aspectos por evaluar), y la segunda, de un modo cualitativo, en la columna reservada para "observaciones". En esta ficha en concreto puede recogerse información de observaciones realizadas hasta en 7 días por semana diferentes. Esto es lo que permite hacer el seguimiento de la evaluación de cada alumno. La forma de realizar la observación de un modo sistemático, pero viable en las condiciones de trabajo habituales de un maestro, es centrarse cada día en un grupo reducido de alumnos (entre 4, y según la capacidad y la práctica desarrollada) y completar la ficha al finalizar la sesión. De esta manera, aproximadamente cada cuatro o cinco sesiones se puede disponer de información sobre todo el alumnado, de un modo mucho más riguroso y más fiable que completar las fichas de observación al final de cada unidad didáctica o cada trimestre.

Ficha de seguimiento individual
Curso académico: $\quad$ Grupo (3 años)
Centro: $\frac{\text { Nombres_ }}{\text { Alumno: Apellidos }}$

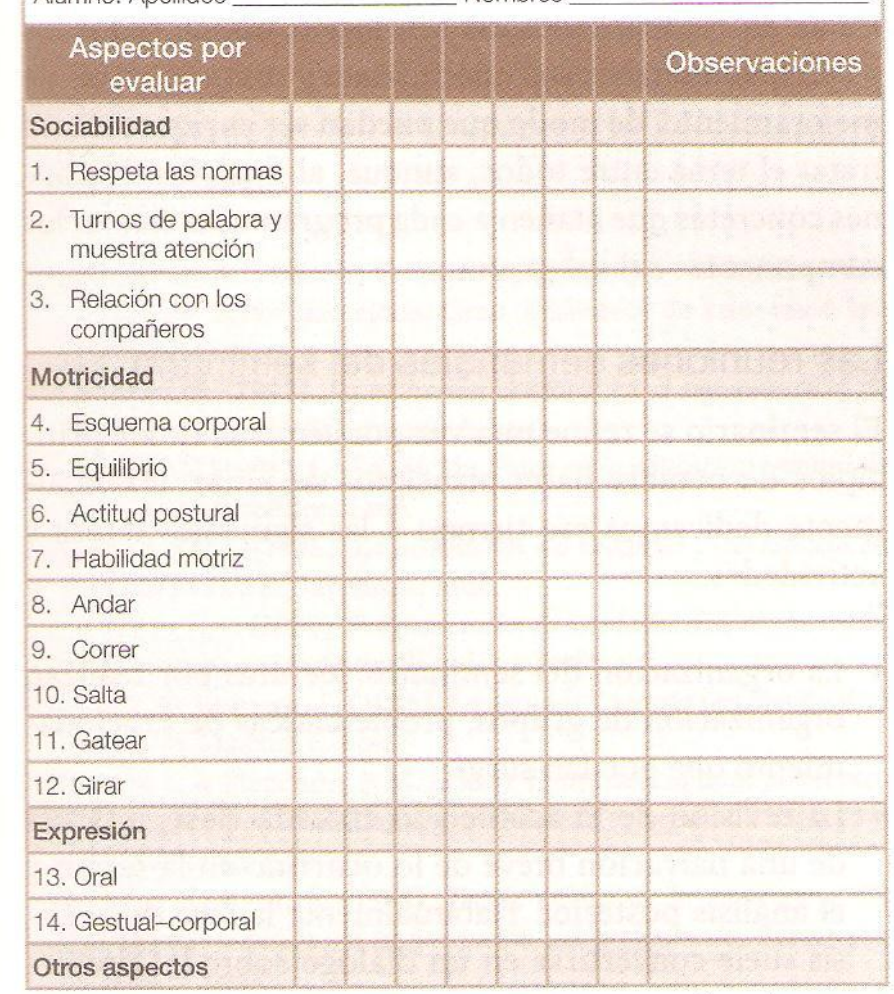

Escala verbal: (1) nada, (2) muy poco, (3) poco, (4) bastante, (5) mucho.

Cuadro 3. Ficha de seguimiento individual genérica utilizada con un grupo de tres años ${ }^{1}$.

1 Basada en las propuestas de López et al. (2004).

\section{Material e instalaciones}

Aunque las instalaciones y el material varían en función de las posibilidades de cada colegio, lo normal es que podamos disponer de un gimnasio o aula multiusos, provistos de colchonetas y cierto material específico (aros, pelotas, bloques de goma-espuma, ladrillos, colchonetas). En algunos centros también disponemos de una pizarra en el gimnasio. En un centro tenemos acceso a un pabellón polideportivo de $20 \times 40 \mathrm{~m}$ y bien equipado. En cambio, en algunas ocasiones no se puede disponer de estas instalaciones y estos materiales, y tenemos que llevar a cabo las sesiones en las aulas normales, y con escaso material (las sillas y las mesas, algún aro y poco más). No es lo más aconsejable, pero tampoco es un impedimento para continuar trabajando.

\section{Un aspecto clave: la organización del Seminario Permanente de Educación Física en Educación Infantil y su metodología de trabajo}

\section{La organización y el funcionamiento del seminario}

El Seminario Permanente de Educación Física en Educación Infantil es la estructura organizativa a través de la cual funcionamos como grupo de trabajo, desarrollando, poniendo en práctica y evaluando este proyecto educativo. El seminario surge a partir de un proyecto de colaboración entre el profesorado de educación infantil de algunos colegios de la ciudad y un grupo de personas interesadas en trabajar la motricidad del alumnado de esta etapa educativa. Tiene su sede en la escuela de magisterio de la ciudad y está compuesto, en su mayor parte, por alumnado de la escuela de Magisterio en periodo de formación inicial, aunque no únicamente. La mayoría de sus miembros pertenece a las especialidades de educación infantil y EF; también hay maestras de la especialidad de primaria y musical.

La estructura básica de funcionamiento consiste en la puesta en práctica de una hora semanal de intervención educativa con el alumnado del segundo ciclo de educación infantil, a cargo de grupos pequeños, normalmente de tres componentes, en forma estable y continua durante todo el curso. También una vez a la semana se lleva a cabo la reunión del seminario con todos los subgrupos, para analizar en conjunto lo acontecido durante las sesiones de la última semana. Como hemos explicado en el apartado anterior, utilizamos una metodología de in- 
vestigación-acción, con la finalidad de ir perfeccionando nuestra intervención educativa, así como la calidad de los procesos de enseñanza-aprendizaje que llevamos a cabo (Carr y Kemmis, 1988; Elliott, 1990, 1993; Latorre, 2003; Kemmis y McTaggart, 1988; Stenhouse, 1987).

$\mathrm{Al}$ comienzo de cada curso, el primer paso es la organización inicial del seminario y el diseño del proyecto curricular por parte de todo el grupo. Mientras llevamos a cabo este proceso, los subgrupos van estableciendo la relación con los centros en que van a trabajar durante el curso, de modo que cada subgrupo trabaje todo el curso con el mismo grupo-aula. Cada equipo comienza a preparar su propio programa de intervención, que luego se presenta y ajusta en las reuniones del seminario. El siguiente paso es la puesta en práctica de dichos programas de intervención, que implica las siguientes tareas: planificación previa de la sesión, preparación de la sala; aclarar y asumir los roles de cada persona, llevar a cabo la sesión, análisis posterior de lo ocurrido en la sesión realizada y la toma de decisiones (replanteamiento) para la próxima sesión. Este análisis lo realiza en un primer momento el grupo de maestras y maestros al finalizar la sesión y se completa en la siguiente reunión del seminario. Por último, se realiza una transcripción de todo ello, se fotocopia y archiva, para posteriores consultas. Este ciclo de funcionamiento se repite durante todo el año.

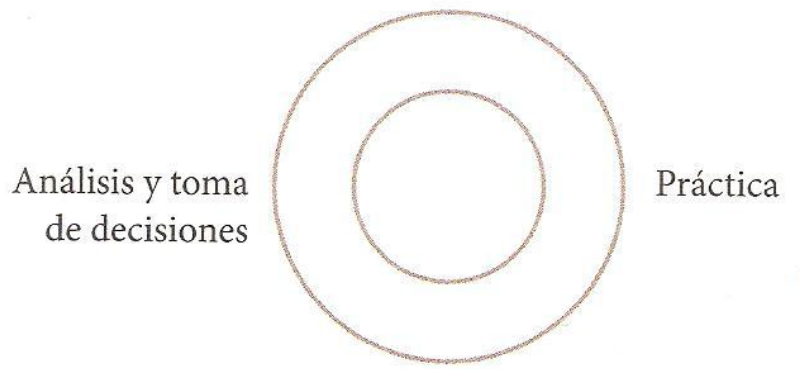

Reflexión

Gráfica 2. Círculos semanales de reflexión sobre la acción que originan las espirales de investigación-acción durante el curso.

\section{La organización de cada subgrupo de trabajo y el trabajo en los centros educativos}

Los subgrupos de trabajo suelen estar compuestos por tres personas, cada una de las cuales desempeña un papel y una función claramente establecidos ("profesor principal", "profesor de apoyo", "observador"). El profesor principal es el responsable de la dirección de la sesión y el principal foco de información para el alumnado. El profesor de apoyo ayuda al profesor principal y realiza apoyos a las niñas y los niños que lo requieren durante la actividad motriz. El observador lleva a cabo la narración de la sesión y la evaluación del alumnado a través de las fichas de observación grupales.

Esta organización permite una mejor intervención docente, la realización de una narración más detallada sobre lo que ocurre en clase, que facilita el análisis reflexivo posterior y un proceso de enseñanza-aprendizaje de mayor calidad. Las tres funciones van rotando durante el curso (normalmente cada trimestre), de modo que las tres personas desempeñan los tres roles durante el curso académico. En algunas ocasiones los subgrupos están compuestos por dos o por cuatro personas, aunque intentamos que sea algo excepcional. Las sesiones se llevan a cabo dentro del horario lectivo y en coordinación con las profesoras de los grupos de educación infantil implicados en esta experiencia educativa que, en algunos casos, asumen uno de los roles. Tienen una periodicidad semanal, en un día y un horario fijos. Se realizan en el gimnasio del centro o en un pabellón polideportivo cercano.

Cada uno de los subgrupos es el responsable de la evolución y la concreción de su programa de intervención: fijan el eje sobre el cual se comienza a trabajar, deciden cómo se hace evolucionar cada eje y cuándo llega el momento de cambiarlo por otro. Todas estas cuestiones se tratan en las reuniones del seminario, donde todos los participantes hacen sus valoraciones y sus propuestas de mejoramiento, de modo que puedan ser enriquecidas al tratar el tema entre todos, aunque, al final, las decisiones concretas que atañen a cada programa las toman los componentes del subgrupo.

\section{Las reuniones semanales del seminario}

El seminario se reúne una vez por semana, desde principios de octubre hasta principios de junio. Habitualmente dedicamos ese tiempo a las siguientes tareas y actividades:

- La organización del seminario: lecturas por realizar, organización de grupos, problemáticas de funcionamiento que puedan surgir.

- La revisión de lo acontecido en cada clase, a través de una narración breve de lo ocurrido en la sesión y el análisis posterior. Habitualmente la fase de análisis suele convertirse en un diálogo sobre las problemáticas y las dificultades que surgen. A partir de ahí se toman decisiones para mejorar la docencia en las próximas sesiones.

- La entrega y la acumulación de transcripciones (narración y análisis) de sesiones anteriores. 
En algunas ocasiones una parte de las reuniones del seminario puede dedicarse también a ampliar el bagaje de recursos didácticos, cuando éstos son necesarios para la práctica y parte de los componentes del grupo no los dominan (canciones motrices, organización de los entornos y rincones para trabajar determinados contenidos, juegos cooperativos sencillos, etcétera).

\section{Conclusiones}

En este artículo hemos presentado de manera sinóptica nuestra forma de trabajar la motricidad en educación infantil. Esta propuesta ha sido desarrollada a través de un seminario permanente que lleva funcionando ocho años, mediante dinámicas de investigación-acción que nos permiten ir mejorando los procesos de enseñanza-aprendizaje en que estamos implicados, a la vez que generamos un proceso de perfeccionamiento docente eminentemente colaborativo. En la introducción hemos realizado un breve análisis del estado de la cuestión en nuestro país y hemos explicado la fundamentación teórica y experimental del proyecto. Después hemos presentado las características principales de la

\section{Referencias}

Aucouturrier, B. y Mende, G. (2004). ¿Por qué los niños y las niñas se mueven tanto? Barcelona: Graó. Biblioteca de Educación Infantil, 1 .

De Andrés, N. (1993). La expresión corporal en el segundo ciclo de educación infantil. Salamanca: Amarú.

Aznar, P. et al. (1998). La Educación Física en la educación infantil de 3 a 6 años. Barcelona: Inde.

Blández Ángel, J. (1995). La utilización del material y del espacio en Educación Física. Barcelona: Inde.

. (2000). Programación de unidades didácticas según ambientes de aprendizaje. Barcelona: Inde.

Blazquez, D. y Ortega, E. (1985). La actividad motriz (en el niño de 3 a 6 años). Madrid: Cincel.

Bueno, M. L. y Manchón, P. M. (1990). Educación infantil por el movimiento corporal. Identidad y autonomía personal, 2o. ciclo (3-6 años). Madrid: Gymnos.

Carr, W. y Kemmis, S. (1988). Teoría crítica de la enseñanza. La investigación-acción en la formación del profesorado. Barcelona: Martínez-Roca.

Desrosiers, P. y Tousignant, J. L. (2005). Psicomotricidad en el aula. Barcelona: Inde.

Elliott, J. (1990). La investigación-acción en educación. Madrid: Morata.

(1993). El cambio educativo desde la investigación-acción. Madrid: Morata. propuesta (objetivos, principios de procedimiento, círculos de aprendizaje reflexivo utilizados, modelo genérico de sesión, sistema e instrumentos de evaluación, contenidos, instalaciones y materiales). Por último, hemos explicado la organización y el funcionamiento del seminario, que es la comunidad de aprendizaje en que se apoya el proceso de intervención e investigación en la práctica educativa. En estos ocho años hemos experimentado el modelo en 30 grupos de educación infantil. En su mayor parte sólo un curso, pero con varios de ellos durante dos o tres cursos seguidos. En todos los casos, esta propuesta ha demostrado su viabilidad y su adecuación para trabajar la motricidad en la etapa de educación infantil en horario lectivo, así como una amplia capacidad para atender la diversidad del alumnado, tanto la diversidad propia de cualquier grupo, como la específica del alumnado con necesidades educativas especiales. En líneas de trabajo posteriores puede ser interesante comprobar si también es viable y adecuada para trabajar en contextos extraescolares, o bien comprobar si pueden generarse propuestas eclécticas, combinando esta propuesta con otro tipo de metodologías.
Escribá, A. et al. (1999). Psicomotricidad. Fundamentos teóricos aplicables a la práctica. Madrid: Gymnos.

Esparza y Petroli. (1984). La psicomotricidad en el jardin de infantes. Buenos Aires: Paidós.

Farreny, M. T. y Román Sánchez, G. (2005). El descubrimiento de sí mismo. Actividades y juegos de motricidad en la escuela infantil, 2o. ciclo. Barcelona: Graó.

Gil Madrona, P. (2003). Diseño y desarrollo curricular en Educación Física y educación infantil. Sevilla: Wanceulen.

Godall, T. y Hospital, A. (2000a). 50 propuestas de actividades para el 2o. ciclo de educación infantil (3-4 años). Barcelona: Paidotribo. . (2000b). 50 propuestas de actividades para el 2o. ciclo de educación infantil (4-5 años). Barcelona: Paidotribo. (2000c). 50 propuestas de actividades para el 2o. ciclo de educación infantil (5-6 años). Barcelona: Paidotribo.

González, L. y Gómez, J. (1989). La Educación Física en la primera infancia. Buenos Aires: Stadium.

González Rodríguez, C. (2001). Educación Física en preescolar. Barcelona: Inde.

Kemmis, S. y McTaggart. (1988). Cómo planificar la investigaciónacción. Barcelona: Laertes.

Kolb, D. (1984). Experiential Learning. New York: Prentice-Hall.

La Torre, A. (2003). La investigación-acción. Conocer y cambiar la práctica educativa. Barcelona: Graó. 
Lapierre, A. (1984). La educación psicomotriz en la escuela maternal. Barcelona: Médica y técnica.

Lapierre, A. y Aucouturrier, B. (1974a). Los contrastes. Barcelona: Científico-Médica.

(1974b). Los matices. Barcelona: Científico-Médica. (1977a). Asociación de contrastes. Barcelona: Científico-Médica.

Médica.

(1977b). Estructuras y ritmos. Barcelona: Científico-

Lázaro Lázaro, A. (2000). Nuevas experiencias en educación psicomotriz. Zaragoza: Mira.

Le Boulch, J. (1969). La educación por el movimiento. Buenos Aires: Paidós.

(1978). Hacia una ciencia del movimiento humano. Buenos Aires: Paidós.

López, V., Monjas, R. y Pérez, D. (2003). Buscando alternativas a la forma de entender y practicar la Educación Física escolar. Barcelona: Inde. via: Diagonal.

(1999). Educación Física, evaluación y reforma. Sego-

(2004). La Educación Física en educación infantil: una propuesta y algunas experiencias. Buenos Aires: Miñó y Dávila.

López Pastor, V. M. et al. (2006). La evaluación en Educación Física. Revisión de los modelos tradicionales y planteamiento de una alternativa: la evaluación formativa y compartida. Buenos Aires: Miñó y Dávila.

Lleixa Arribas, T. (1991). La Educación Física en preescolar y ciclo inicial (4 a 8 años). Barcelona: Paidotribo. (2001). Juegos sensoriales y de conocimiento corporal. Barcelona: Paidotribo.

Martínez, P., García, M. C. y Montoro, J. M. (1988). Primeros pasos en psicomotricidad. Madrid: Narcea.

Martínez, P. y Núñez, J. (1978). Psicomotricidad y educación preescolar. Madrid: Nuestra Cultura.
Mendiara Rivas, J. (1997). Educación Física y aprendizajes tempranos. Contribución al desarrollo global de los niños de 3 a 6 años y estudio de sus estrategias de aprendizaje en espacios de acción y aventura. Tesis doctoral inédita. Universidad de Zaragoza. . (1999). Espacios de acción y aventura. Apunts: Educación Física y Deportes, 56. 65-70.

Mendiara, J. y Gil, P. (2003). Psicomotricidad. Evolución, corrientes y tendencias actuales. Sevilla: Wanceulen.

Miraflores, E. et al. (2006). El esquema corporal (240 juegos). Madrid: CCS.

Pastor Pradillo, J. L. (2002). Fundamentación conceptual de una intervención psicomotriz en Educación Física. Barcelona: Inde.

Rigal, R. (2006). Educación motriz y educación psicomotriz en preescolar y primaria. Barcelona: Inde.

Santiago, P. (1985). De la expresión corporal a la comunicación interpersonal. Teoría y práctica de un programa. Madrid: Narcea.

Stenhouse, L. (1987). La investigación como base de la enseñanza. Madrid: Morata.

Stokoe, P. y Harf, R. (1987). La expresión corporal en el jardín de infantes. Barcelona: Paidós.

Vaca Escribano, M. (1995). El tratamiento pedagógico de lo corporal en educación infantil. Tesis doctoral inédita. Madrid: UNED. (2000). Reflexiones en torno a las posibilidades educativas del tratamiento pedagógico de lo corporal en el segundo ciclo de educación infantil. Revista Interuniversitaria de Formación del Profesorado, 37, 103-120. Zaragoza: AUFOP.

Vayer, P. (1983). El diálogo corporal. Acción educativa en el niño de 2 a 5 años. Barcelona. Científico-Médica. co-Médica. (1983). El niño frente al mundo. Barcelona: Científi-

Vila, B. y Cardo, J. I. (2005). Material sensorial (0-3 años). Manipulación y experimentación. Barcelona: Graó. Biblioteca Infantil, 8. 